\title{
The SBND experiment
}

\author{
Serhan Tufanli*t \\ Yale University, \\ New Haven, CT 06520 - USA \\ E-mail: serhan.tufanli@yale.edu
}

The Short-Baseline Near Detector (SBND) is one of the three Liquid Argon Time Projection Chamber (LArTPC) experiments to be situated along the Booster Neutrino Beam (BNB) to form Short-Baseline Neutrino (SBN) program at Fermilab. The detector will be situated at $110 \mathrm{~m}$ far from the BNB proton target, with $112 \mathrm{t}$ active volume, to perform precise measurement of neutrino cross-sections in the energy range of $0.5-1 \mathrm{GeV}$. These measurements are necessary to do a high-sensitivity search for light $\left(\sim 0.1-10 \mathrm{eV}^{2}\right)$ sterile neutrino oscillations. In addition to physics goals, LArTPC technology developed and used in SBND will form an important step towards the future long-baseline neutrino experiments. This paper will discuss the details of SBND experiment and its current status.

XIII International Conference on Heavy Quarks and Leptons

22- 27 May, 2016

Blacksburg, Virginia, USA

\footnotetext{
* Speaker.

${ }^{\dagger}$ On behalf of the SBND Collaboration.
} 


\section{Introduction}

From 1989 to 1995, ALEPH, DELPHI, L3, OPAL experiments at the Large Electron-Positron collider (LEP) at CERN and over a similar period, the SLD experiment at SLAC Linear Collider verified the existence of three active neutrino flavors [1]. In the last two decades, measurements from neutrino oscillation experiments have proved mixing and oscillation among these three active flavors $[2,3]$. However, not all the experimental data fits the three flavor oscillation phenomena. Electron like excess from the LSND [4] and the MiniBooNE experiments [5], or $\bar{v}_{e}$ disappearance from nuclear reactors and radioactive sources [6] can not be explained with the standard three flavor oscillations and require the existence of sterile neutrino with a mass range of $1 \mathrm{eV}$. These anomalies taken by themselves are not strong indicator of sterile neutrinos. However, all are pointing in the same direction and combined effect can be a hint for the new physics.

The Fermilab Short-Baseline Neutrino program (SBN) [7] was designed to provide a definitive answer to whether the short-baseline anomalies can be associated with high $\Delta \mathrm{m}^{2}$ sterile neutrino oscillations. SBN is a three detector system with a new near detector, SBND, MicroBooNE and a large far detector, ICARUS. These detectors will sit on-axis in the Booster Neutrino Beam (BNB), which has $0.7 \mathrm{GeV}$ neutrino energy peak and well known $0.5 \% v_{e}$ contamination. As shown in the schematic view of SBN in Figure 1, SBND will be situated at $110 \mathrm{~m}$ from the BNB target, followed by MicroBooNE at $470 \mathrm{~m}$ and ICARUS be at $600 \mathrm{~m}$.

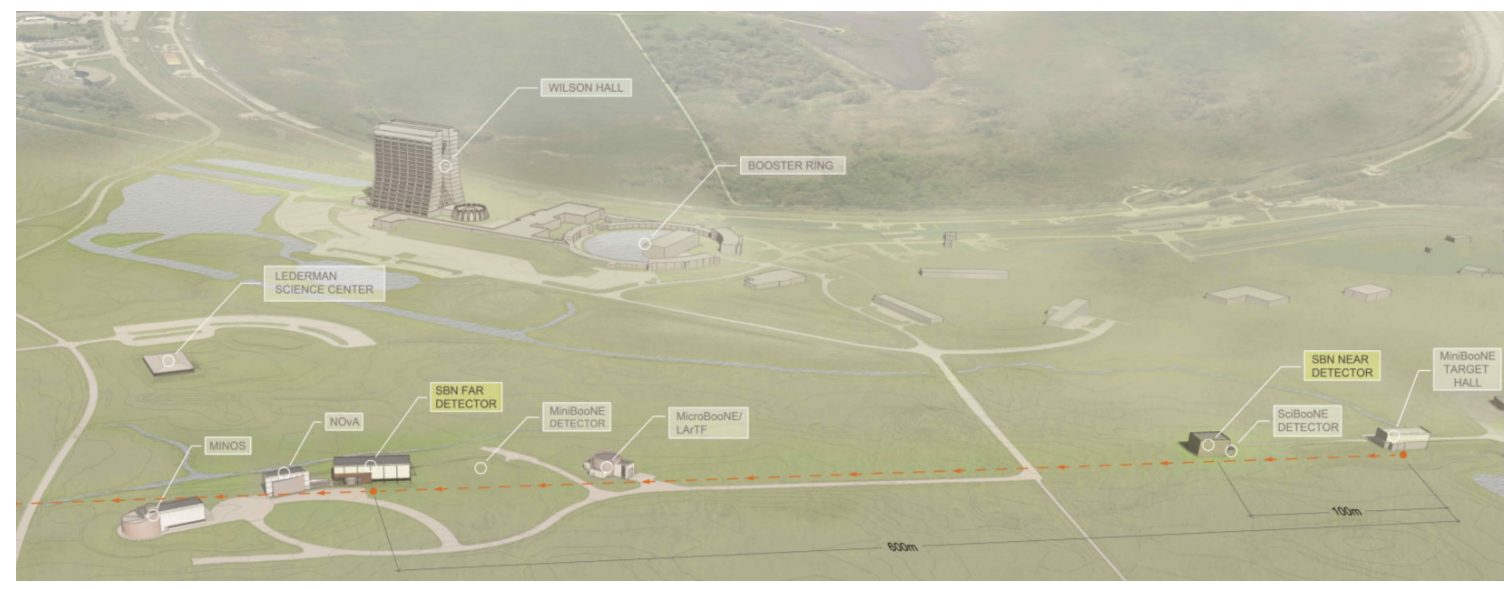

Figure 1: Schematic view of the SBN complex at Fermilab.

Possible existence of sterile neutrinos will be studied by electron neutrino appearance and muon neutrino disappearance using the Liquid Argon Time Projection Chamber (LArTPC) technology [8]. LArTPC provides millimetric spatial resolution and precise measurement of calorimetric information which help to reduce the neutral-current induced photon production background, the dominant background source for electron neutrino experiments. For the electron neutrino appearance, combining 3 experiments, SBN will cover $99 \%$ of the LSND allowed region with more than $5 \sigma$ significance with three years of run. In the case of muon neutrino disappearance, SBN will extend the search by an order of magnitude beyond the combined analysis of SciBooNE and MiniBooNE [9]. Figure 2 shows the SBN sensitivities both for electron neutrino appearance and muon neutrino disappearance. 

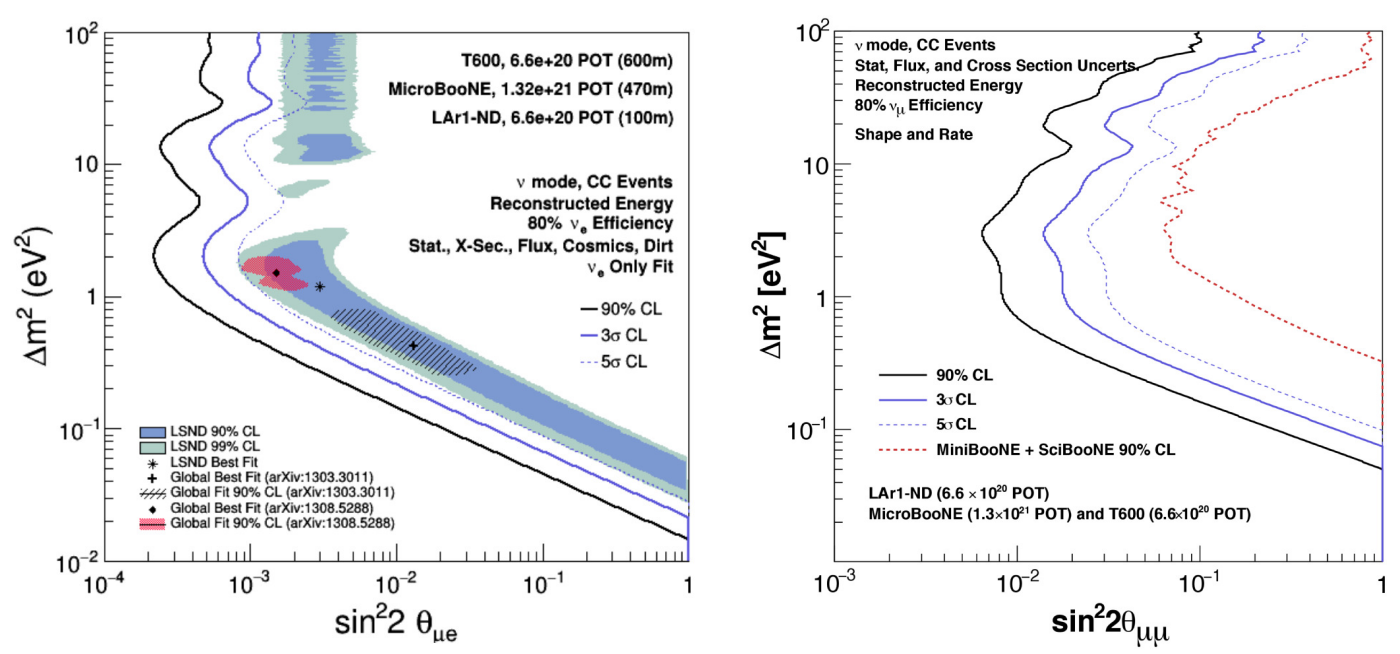

Figure 2: Sensitivities for electron neutrino appearance (left) and muon neutrino disappearance (right).

Precise measurement of $v$-Ar nucleus cross-section is crucial for the correct interpretation of the experimental results, including sterile neutrino studies and the future liquid argon long-baseline physics goals. SBN provides an ideal venue to conduct precision cross section measurements in the sub-GeV and few $\mathrm{GeV}$ range. Especially SBND will collect more than 2 million neutrino interactions per year assuming $2.2 \times 10^{20}$ protons on target, 1.5 million of which will be muon neutrino charged current interactions, 12,000 electron neutrino charged current interactions, 250,000 neutral current interactions and a few hundred elastic scattering events. In addition to low energy BNB neutrinos, off-axis neutrinos from Main Injector, or NuMi [10], will provide cross section studies for the different energy region. With its 476 ton of active volume, ICARUS is expected to detect 100,000 events per year originating from NuMi beam.

\section{The SBND Experiment}

The SBND experiment is an international collaboration with 170 members from 20 US and 12 international institutions. The main purpose of the experiment is to provide detailed characterization of the BNB beam before possible oscillation and to study $v$-Ar cross sections with a large data sample. In addition to the physics goals, the detector technology developed and used in this experiment will be an important step towards the future detectors for long-baseline neutrino experiment.

SBND has designed based on incorporated experience from the ICARUS and MicroBooNE experiments. The main component of the detector will be a Time Projection Chamber (TPC) placed inside a membrane cryostat. The detector will be situated in a new building, located adjacent to the SciBooNE hall as shown in Figure 1. The conceptual design of the SBND cryostat and TPC in it is shown in Figure 3.

\subsection{SBND Time Projection Chamber}

The SBND TPC will have $5 \times 4 \times 4 \mathrm{~m}^{3}$ volume, with two $2 \mathrm{~m}$ drift regions. The central cathode assembly plane (CPA) will separate the drift volumes. Charge produced by the ionizing particles 


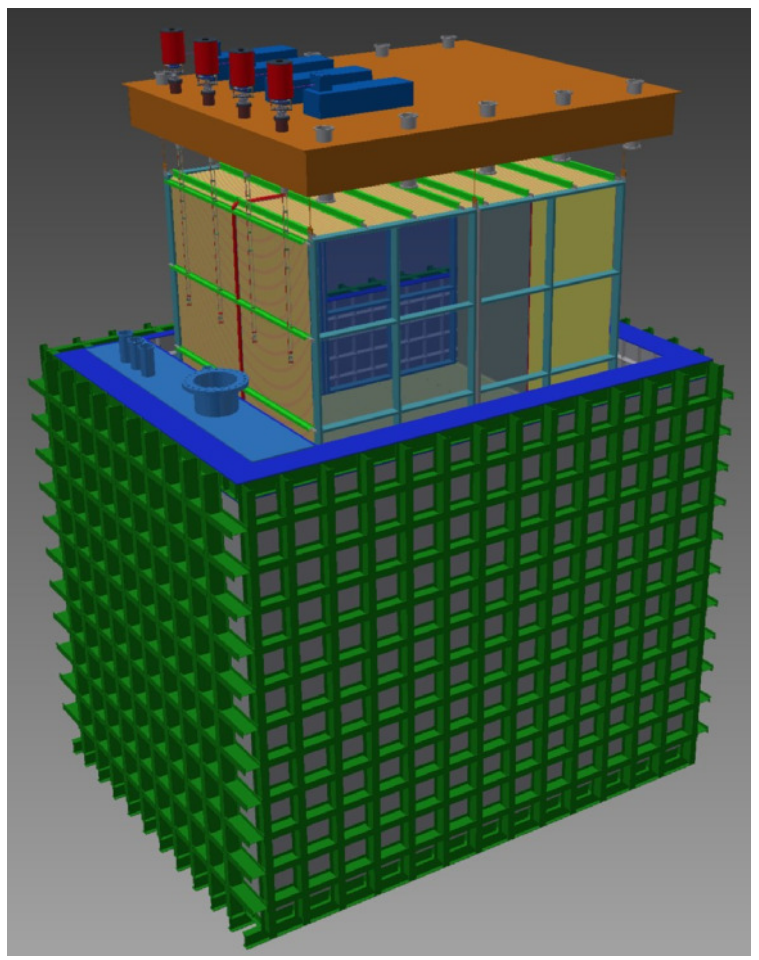

Figure 3: Conceptual design of the SBND membrane cryostat, TPC and the removable top flange.

will be drifted from CPA to wires planes mounted on the two anode assembly planes (APA) at either end of the TPC. Each APA will have $2.5 \mathrm{~m} \times 4 \mathrm{~m}$ dimensions and able to hold three wire planes; a vertical collection plane and two induction planes at $\pm 60^{\circ}$ angles to the vertical. Each wire plane will have a $3 \mathrm{~mm}$ wire pitch, identical to the ICARUS and MicroBooNE in order to have similar electron/photon separation efficiencies. Wires have $150 \mu \mathrm{m}$ diameter and are made of Copper-Beryllium. In total 11,264 wires will be connected to the wire boards, which provides connection to readout electronics, with epoxy and electrically terminated solder.

The CPA design will have the same dimensions as APA and consist of a stainless-steel structure. The surface of the CPA panels will be highly transparent mesh plane to allow more efficient light collection. CPA surface will be electrically polished in order to avoid any sharp edges that could lead to electric discharges. Design model of the SBND time projection chamber is shown in Figure 4.

Electric field inside the drift volume is a key parameter for successful TPC operation. To achieve $500 \mathrm{~V} / \mathrm{cm}$ drift field strength, SBND requires $-100 \mathrm{kV}$ bias on the CPA frame. $-100 \mathrm{kV}$ is generated by a Heinzinger PNChp power supply ${ }^{1}$ and delivered to the high voltage feed-through (HV-FT) by a high voltage cable. HV-FT brings the voltage bias inside to the cryostat and delivers it to the CPA. The HV-FT has a coaxial design consisting of an inner stainless-steel conductor and outer stainless-steel grounding tube with a ultra-high molecular-weight polyethylene (UHMW-PE) insulator tube interleaved between. UHMW-PE has a diameter of $6.3 \mathrm{~cm}$ in order to reduce electric field density below $30 \mathrm{kV} / \mathrm{cm}$, which is a conservative required design value to avoid electric dis-

\footnotetext{
${ }^{1}$ www.heinzinger.com
} 


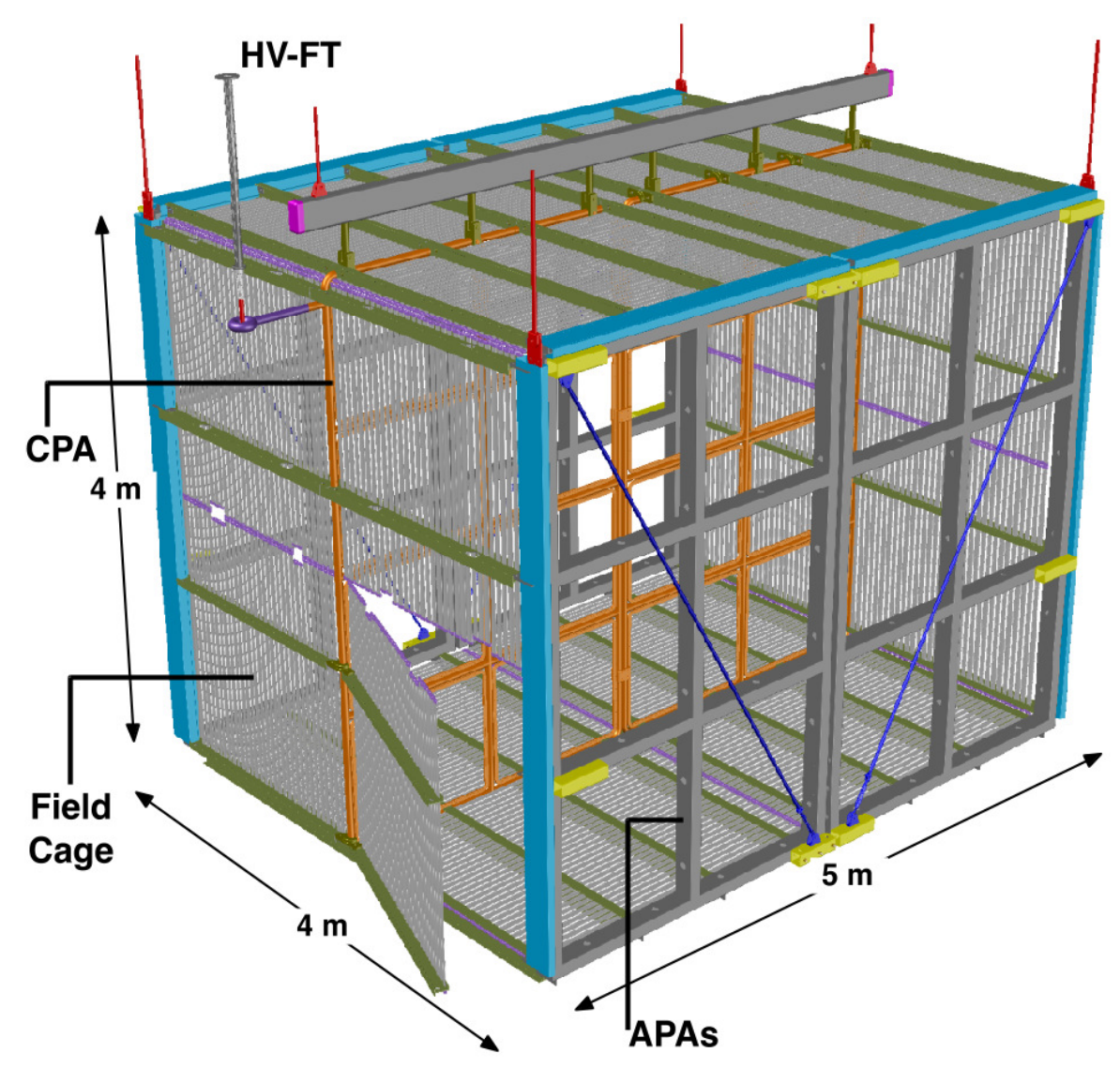

Figure 4: Design of the SBND time projection chamber.

charges in liquid argon. The length of the HV-FT is $\sim 1.8 \mathrm{~m}$ and the bottom of the inner conductor has a spring-loaded ball contact which mates to a donut-shaped receptacle attached to the CPA to provide bias to the cathode. The spring loaded mechanism allows for small displacements of the HV-FT due to the thermal contractions of the cryostat (from which the HV-FT is mounted) without loss of HV-FT to CPA contact.

The uniformity of the $500 \mathrm{~V} / \mathrm{cm}$ drift field over the TPC active volume is provided by field cage rings. In each drift volume, 33 stainless steel rolled profiles and voltage divider on them gradually brings the voltage from $-100 \mathrm{kV}$ on CPA up to ground voltage. Even if the TPC has field cage for the uniform field, deviations of the field from perfect uniformity may arise due to accumulation of positive argon ions in the drift volume. In order to measure and correct electric field non-uniformities, a pulsed UV laser, using a $\lambda=266 \mathrm{~nm}$ source to produce ionization, will be mounted on the four corners of the TPC.

TPC readout is comprised of three parts: cold electronics, warm interface electronics and signal feed-through. The signal from each wire is pre-amplified and shaped by a CMOS analog front end ASIC, then digitized by a CMOS ADC ASIC inside the cryostat. The digitized signal is sent to an FPGA, which aggregates data from multiple ADC chips and multiplexes it to high speed serial links. The serial data is then sent over cold cables through a feed-through to the warm interface board installed outside of the cryostat on top of the signal feed-through. The warm 
interface board receives the electrical serial data from the cold electronics and converts it to optical signals for transmission over a fiber optical link to the TPC readout module, housed in a crate. Once the signal arrives at the TPC readout module, it is processed in an FPGA for compression, reduction, and storage. Processed data is buffered on board temporarily and then transmitted to DAQ PCs through the crate backplane and optical links. Data received on PCs is then stored in hard drives for further analyses.

\subsection{Light Detection and Cosmic Ray Tagging Systems}

SBND provides an excellent test-bed for light collection system in a LAr detector. The opportunity to test new approaches side by side is an important benefit for the goal of informing an optimized design for use in future LAr detectors for long-baseline neutrino physics. The SBDN photon detection system will be placed on the APA's, behind the wire planes and will be composed of 144 8" Hamamatsu cryogenic PMTs and 96 light guide bars. Each light guide bars will have $1.15 \times 0.084 \mathrm{~m}^{2}$ area and will be readout by SiPMs. Both PMTs and light guide bars will be coated with TetraPhenil-Butadiene wavelength shifter to convert incident $128 \mathrm{~nm}$ light to $430 \mathrm{~nm}$ light, which can be efficiently readout.

Cosmic ray muons are the most abundant background in a LArTPC at surface. Muons do not contribute significantly to the background for the sterile neutrino search but they produce $\delta$ rays, which in turn produce photons by Bremsstrahlung and they could possibly mimic a $v_{e}$-like interaction signature. In order to mitigate the cosmic ray background, a cosmic ray tagger, that detects cosmic muons, measures their time and position relative to beam events will be installed around the cryostat. The SBND cosmic ray tagger will be composed of scintillating planes, each consisting $1 \times 10 \times 400 \mathrm{~cm}$ scintillating bars.

\section{Summary and Current Status of SBND}

SBDN experiment has exciting physics capabilities as a near detector in the SBN program. In addition, it provides a great opportunity for detector development towards the future large liquid argon detectors for long-baseline neutrino experiments.

Currently, there are many R\&D and design activities are ongoing in SBND. Design and prototyping activities are progressing and a few key detector components are already in fabrication. Detector installation will take place in 2017 and commissioning will begin in 2018. First beam data is expected to be collected at the end of 2018. Detector site construction is ongoing and will be finalized at the end of 2016. Figure 5 shows the recent status of the detector hall construction at Fermilab.

Even if the proposed schedule for the SBND experiment is a very aggressive one, the collaboration is building on the experience of the design, construction, and operation of existing liquid argon TPCs, and this gives the project the potential to move forward quickly and to attain its goals. 


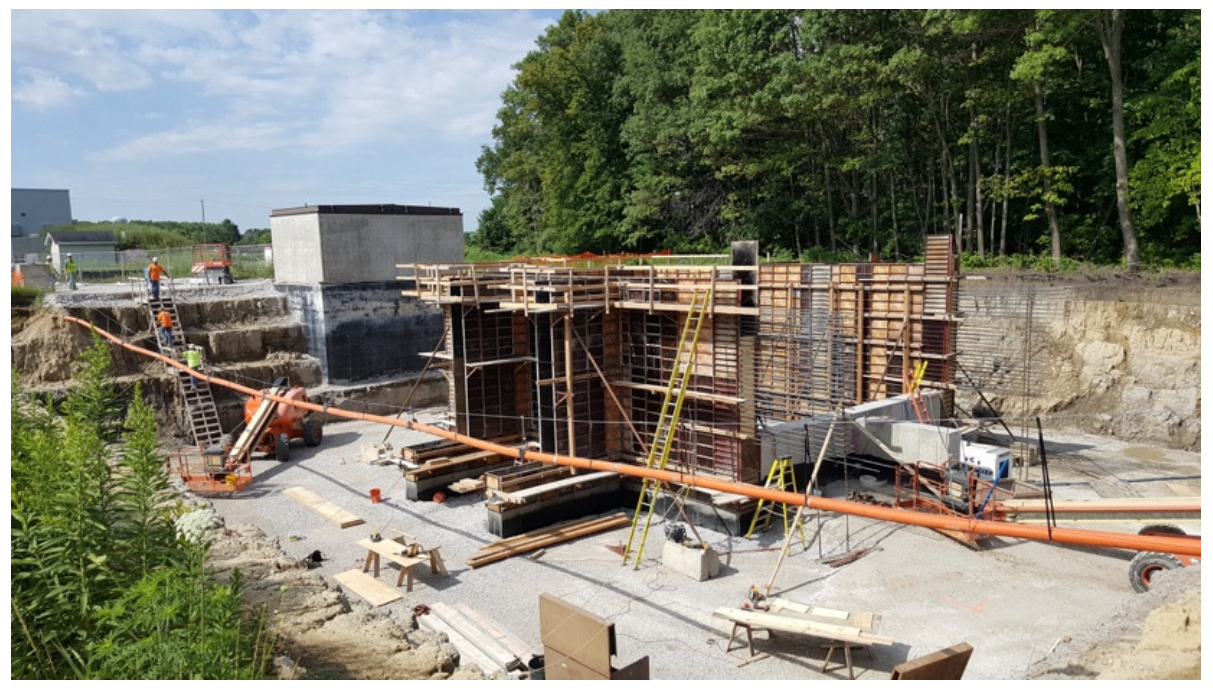

Figure 5: Current status of detector hall construction.

\section{References}

[1] The ALEPH Collaboration, The DELPHI Collaboration, The L3 Collaboration, The OPAL Collaboration, The SLD Collaboration, The LEP Electroweak Working Group, The SLD Electroweak and Heavy Flavour Groups, Precision electroweak measurements on the Z resonance, Phys.Rept. 427 (2006) 257-454 [hep-ex/0509008].

[2] The Super-Kamiokande Collaboration, Evidence for oscillation of atmospheric neutrinos, Phys.Rev.Lett.81 (1998) 1562-1567 [hep-ex/9807003].

[3] The SNO Collaboration, Direct Evidence for Neutrino Flavor Transformation from Neutral-Current Interactions in the Sudbury Neutrino Observatory, Phys.Rev.Lett.89 (2002) 011301 [hep-ex/9807003].

[4] The LSND Collaboration, Evidence for neutrino oscillations from the observation of $\bar{v}_{e}$ appearance in a $\bar{v}_{\mu}$ beam, Phys.Rev.D64 (2001) 112007 [hep-ex/ 0104049 ].

[5] The MiniBooNE Collaboration, Improved Search for $\bar{v}_{\mu} \rightarrow \bar{v}_{e}$ Oscillations in the MiniBooNE Experiment, Phys.Rev.Lett.110 (2013) 161801.

[6] C. Giunti, M. Laveder, Y. F. Li, Q. Y. Liu, and H. W. Long, Update of short-baseline electron neutrino and antineutrino disappearance, Phys.Rev.D86 (2012) 113014 [hep-ph/1210.5715v2].

[7] R.Acciarri et. al, A Proposal for a Three Detector Short-Baseline Neutrino Oscillation Program in the Fermilab Booster Neutrino Beam, [hep . ins-det/1503.01520].

[8] C. Rubbia, The Liquid Argon Time Projection Chamber: A New Concept for Neutrino Detectors, CERN-EP-INT-77-08(1977).

[9] The MiniBooNE Collaboration, The SciBooNE Collaboration, Dual baseline search for muon antineutrino disappearance at $0.1 \mathrm{eV}^{2}<\Delta \mathrm{m}^{2}<100 \mathrm{eV}^{2}$, Phys.Rev.D 86 (2012) 052009, [hep-ex/1208.0322].

[10] P.Adamson et. al, The NuMi neutrino beam, Nucl. Instr. Meth. Phys. Res. A 806 (2016) 279-306, [physics.acc-ph/1507.06690]. 